\title{
Permittivity of SiC ceramic matrix compositesin Ku band
}

\author{
Youssef Ouhassan ${ }^{1 *}$, SeddikBri ${ }^{2}$, My Chrif El boubakraoui ${ }^{3}$, and Mohamed Habibi ${ }^{1}$ \\ ${ }^{1}$ Laboratory of Electronic Systems, Information Processing, Mechanics and Energy, Faculty of Sciences, Ibn Tofail University, \\ Kenetra, Morocco \\ ${ }^{2}$ Materials and Instrumentations group, Superior School of Technology, Moulay Ismail University, Meknes, Morocco \\ ${ }^{3}$ Electronics Instrumentation and physical Measurement, Faculty of Sciences and Technics,Moulay Ismail University, Errachidia, \\ Morocco
}

\begin{abstract}
The objective of this work is to determine the dielectric permittivity of two $\mathrm{SiC}$ ceramic matrix composites. These composites are reinforced with NicalonSiCfibers and $\mathrm{SCS}_{6} \mathrm{SiC}$ fibers $\left(\mathrm{SiC}_{\mathrm{f}} / \mathrm{SiC}\right)$ and have different volume fractions. The results obtained show that the dielectric property depends on the volume fraction and the frequency. Composites with high volume fractions have better dielectric properties than others. The values of the real and imaginary part of the complex permittivity decrease with frequency increase in the Ku-band. Moreover, the imaginary part takes negative values.
\end{abstract}

\footnotetext{
* Corresponding author:ouhassan.youssef@gmail.com
} 


\section{Introduction}

The knowledge of the dielectric permittivity of composite materials is important for several applications $\left[\begin{array}{lll}1, & 2, & 3\end{array}\right], \quad$ particularly in telecommunications, industry and the design of radarabsorbing materials (RAM) [4]. SiC Ceramic matrix composites reinforced by silicon carbide SiCfibers, such as Nicalonfibers, have become important in various applications [5]. They are used for the reinforcement of composite materials in many practical applications and for microwave absorption at high temperature $[6,7]$.

In the literature, several methods have been used to determine the complex permittivity of materials [1]. The Transmission/Reflection (T/R) technique is one of the most adopted methods to characterize materials in the wide frequency range [8]. This technique is based on the use of rectangular waveguides.

This work aims to characterize two ceramic composites with NicalonSiCfiber and $\mathrm{SCS}_{6} \mathrm{SiC}$ fiber in the $\mathrm{Ku}$ band. The goal is to determine the complex permittivity for three volume fractions of these composites. This study is based on the theory of transmission lines and the extraction of $\mathrm{S}_{\mathrm{ij}}$ parameters from the sample inserted inside a rectangular waveguide. The complex permittivity is determined from the $S_{\mathrm{ij}}$ parameters of the sample obtained, using the Nicolson-Ross and Weir (NRW) algorithm [9].

\section{Theory and method}

The microwave technique adopted in this work is a transmission/reflection (T/R) characterization [10]. The complex permittivity is extracted by this technique from the calculated $S_{11}$ reflection coefficient and $S_{21}$ transmission coefficient. The Nicholson-Ross and Weir (NRW) method [9] is fast, direct and applicable to waveguides as well as coaxial lines [11]. This method has been used to determine both the permittivity and permeability of the material studied [12], it is wide frequency band and non-iterative. The sample of the material under test (MUT) is placed in a sample port of a rectangular waveguide WR-62 (Figure.1). The dominant fundamental mode $\left(\mathrm{TE}_{10}\right)$ is considered the only one thatpropagates inside the waveguide.

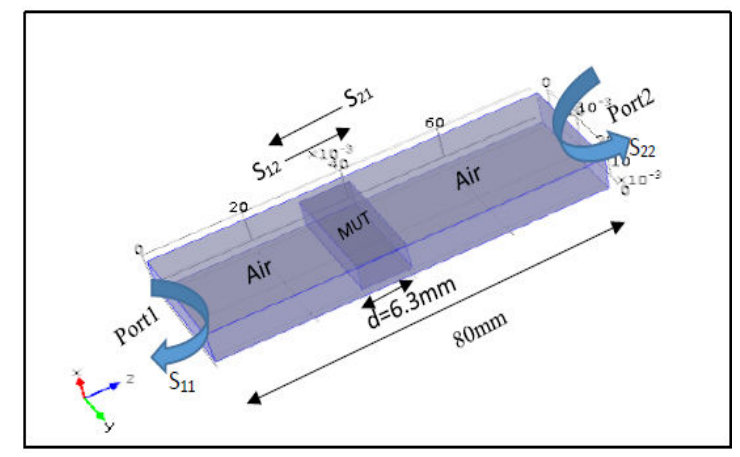

Fig. 1. Schematic figure of the rectangular waveguide configuration used for extraction of the material parameters in the Ku-band.
The following equations established by Weir [13], Ross and Nicolson [9]allow to link the parameters $\mathrm{S}_{11}$ (scattering parameter related to radiation emission from port 1 and collected in port 1) and $S_{21}$ (scattering parameter related to radiation emission from port 1 and collected in port 2) to the reflection and transmission coefficients $\Gamma$ and T, respectively [14]:

$$
\begin{aligned}
& S_{11}=\frac{\Gamma\left(1-\mathrm{T}^{2}\right)}{1-\Gamma^{2} \mathrm{~T}^{2}} \\
& S_{21}=\frac{\mathrm{T}\left(1-\Gamma^{2}\right)}{1-\Gamma^{2} \mathrm{~T}^{2}}
\end{aligned}
$$

The reflection coefficient $\Gamma$ and the transmission coefficient $\mathrm{T}$ are deduced from the $\mathrm{S}_{\mathrm{ij}}$ parameters by the following equations:

$$
\begin{aligned}
& \Gamma=K \pm \sqrt{K^{2}-1} \\
& \mathrm{~T}=\frac{S_{11}+S_{21}-\Gamma}{1-\left(S_{11}+S_{21}\right) \Gamma}
\end{aligned}
$$

with:

$$
K=\frac{S_{11}{ }^{2}-S_{21}{ }^{2}+1}{2 S_{11}}
$$

In the expression of $\Gamma$, the sign must correspond to a physical existence of the reflection coefficient such that $|\Gamma|<1$.The relative permeability of the sample is given by equation (6):

$$
\mu_{r}=\frac{\lambda_{0 g}(1+\Gamma)}{\Lambda(1-\Lambda)}
$$

$\lambda_{0 g}$ is the wavelength guided in the empty cell defined by the following expression :

$$
\lambda_{0 g}=\frac{1}{\sqrt{\frac{1}{\lambda_{0}}-\frac{1}{\lambda_{c}}}}
$$

where $\lambda_{0}$ and $\lambda_{C}$, respectively represent the free space wavelength and the cut-off wavelength. $\Lambda$ is the normalized wavelength related to the transmission coefficient by the relation:

$$
\frac{1}{\Lambda^{2}}=\left[\frac{j}{2 \pi d} \ln (\mathrm{T})\right]^{2}
$$

with $d$ the length of the sample to be characterized.

The relative permittivity is given as a function of the magnetic permeability by the following equations:

$$
\varepsilon_{r}=\frac{\lambda_{0}^{2}}{\mu_{r}}\left(\frac{1}{\Lambda^{2}}+\frac{1}{\lambda_{c}^{2}}\right)
$$

The dielectric dissipation factor or dielectric losses is equal to the ratio between the imaginary part and the real part of the complex dielectric permittivity, its expression is given by: 


$$
\tan \delta=\frac{\varepsilon^{\prime \prime}}{\varepsilon^{\prime}}
$$

To better understand the method presented, the flowchart shown in Figure 2 summarizes the process to be followed to extract the complex permittivity from the sample to be studied:

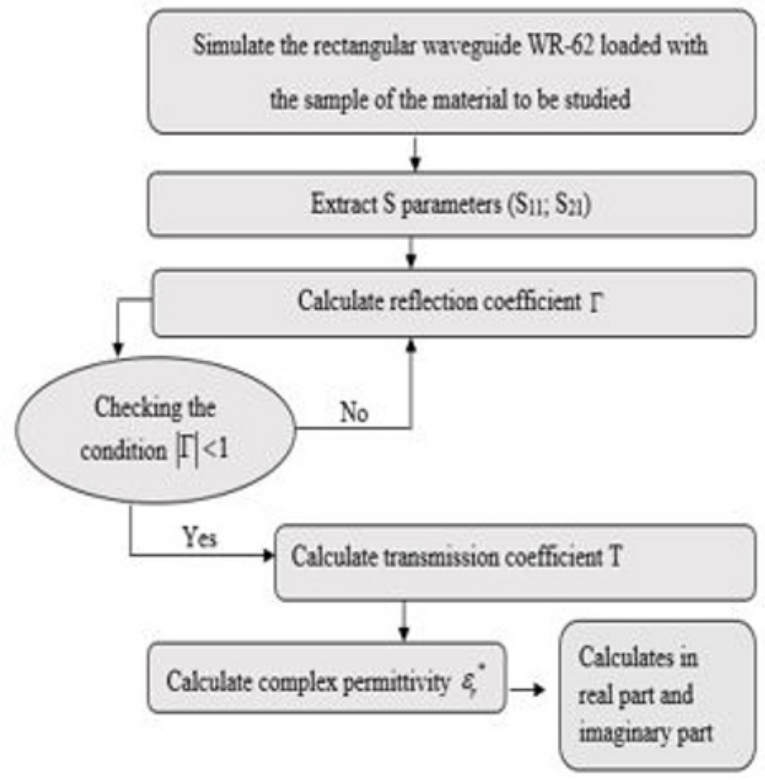

Fig. 2. The flowchart of calculating the complex permittivity by the Nicolson - Ross - Weir method

\section{Results and discussion}

Figures 3 and 4 represent the variation of the parameters $S_{11}$ and $S_{21}$ depending on the frequency in the $\mathrm{Ku}$ band. Figure 3 corresponds to the case of the composite with Nicalon $\mathrm{SiC}$ fiber and figure 4 corresponds to the case of the composite with $\mathrm{SCS}_{6} \mathrm{SiC}$ fiber. The three volume fractions of the composites studied are $f_{1}=0.78 \%, f_{2}=31.4 \%$ and $f_{3}=71.54 \%$.

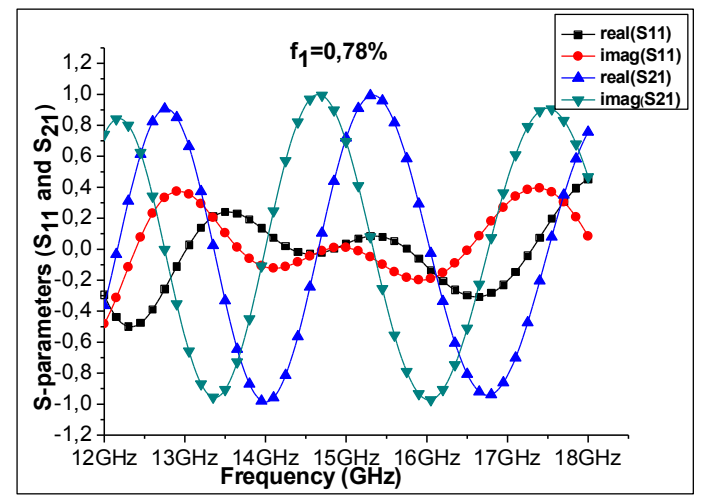

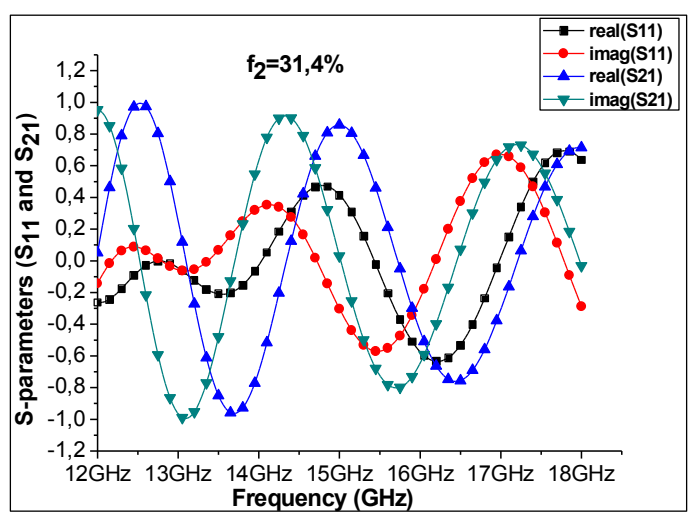

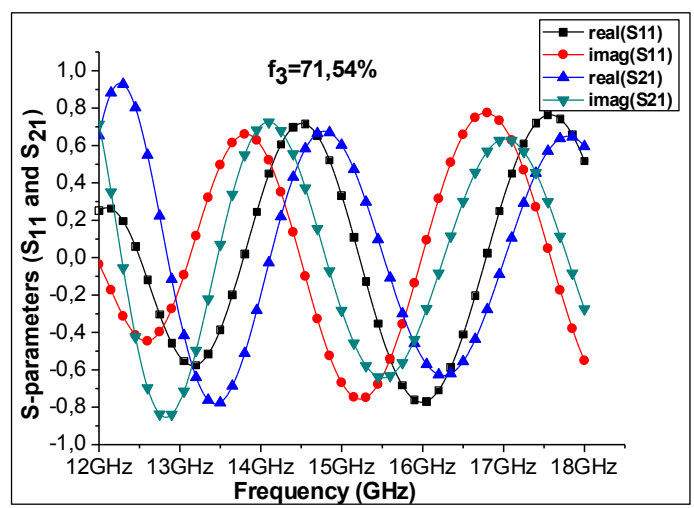

Fig. 3. The parameters $S_{11}$ and $S_{21}$ of composite with Nicalon $\mathrm{SiC}$ fiber in the Ku band.

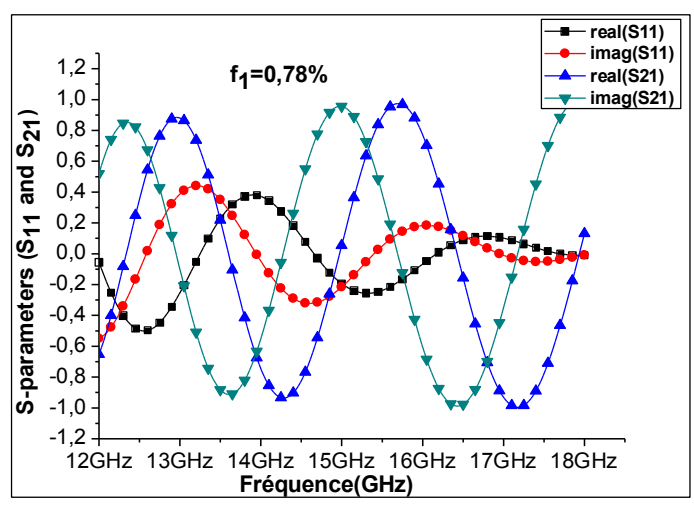



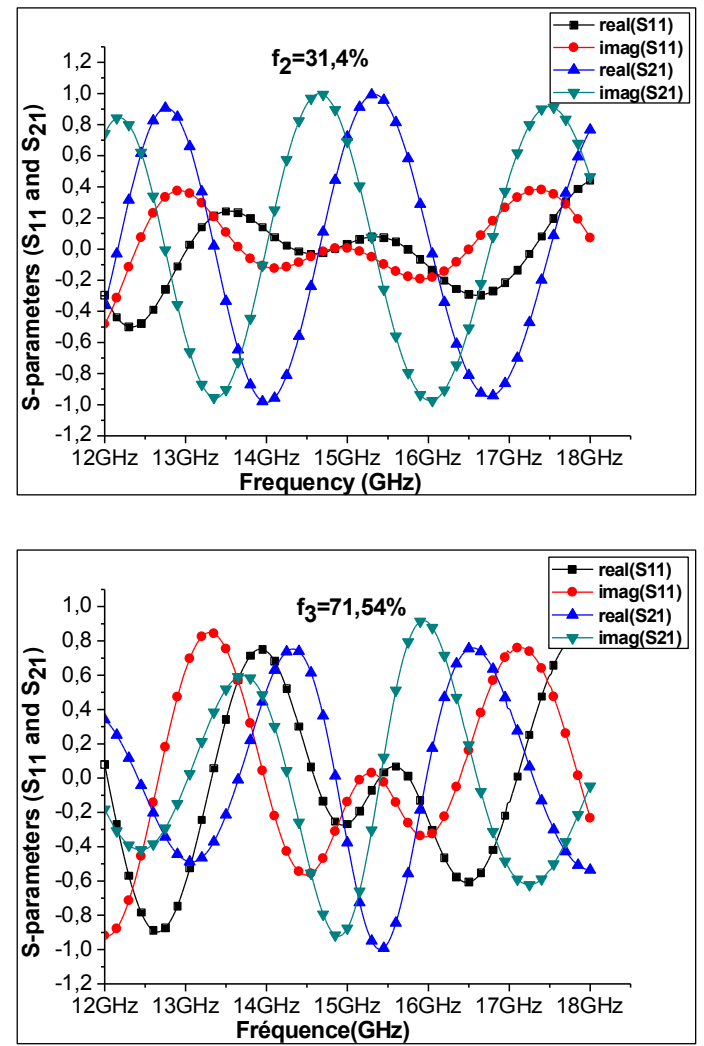

Fig. 4. The parameters $S_{11}$ and $S_{21}$ of composite with $\mathrm{SCS}_{6} \mathrm{SiC}$ fiber in the $\mathrm{Ku}$ band.

The spectra of the parameters $S_{11}$ and $S_{21}$ show the frequency dependence of the reflection parameter $S_{11}$ taken at different filling rates of the inclusions for the composites. We observe that the curve varies, remarkably, when the volume fraction increases from $0.78 \%$ to $71.54 \%$. A comparison between the transmission spectra indicates that the filling rates of the inclusions has a significant impact on the intensity of $\mathrm{S}_{21}$.

Figures 5 and 6 illustrate the variation of the real and imaginary parts of the complex permittivity of the composites studied as a function of the frequency in the $\mathrm{Ku}$ band for the three preceding volume fractions.
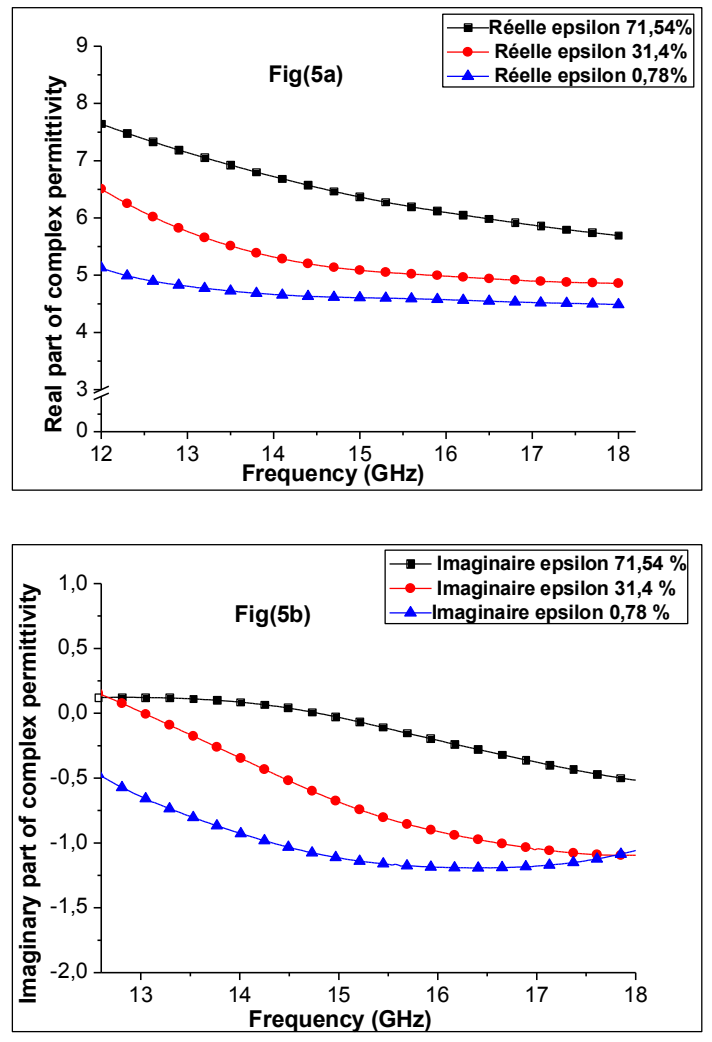

Fig. 5. Evolution of the real (Fig 5a) and imaginary (Fig 5b) parts of the complex permittivity of the composite with Nicalon $\mathrm{SiC}$ fiber as a function of the frequency for three volume fractions.
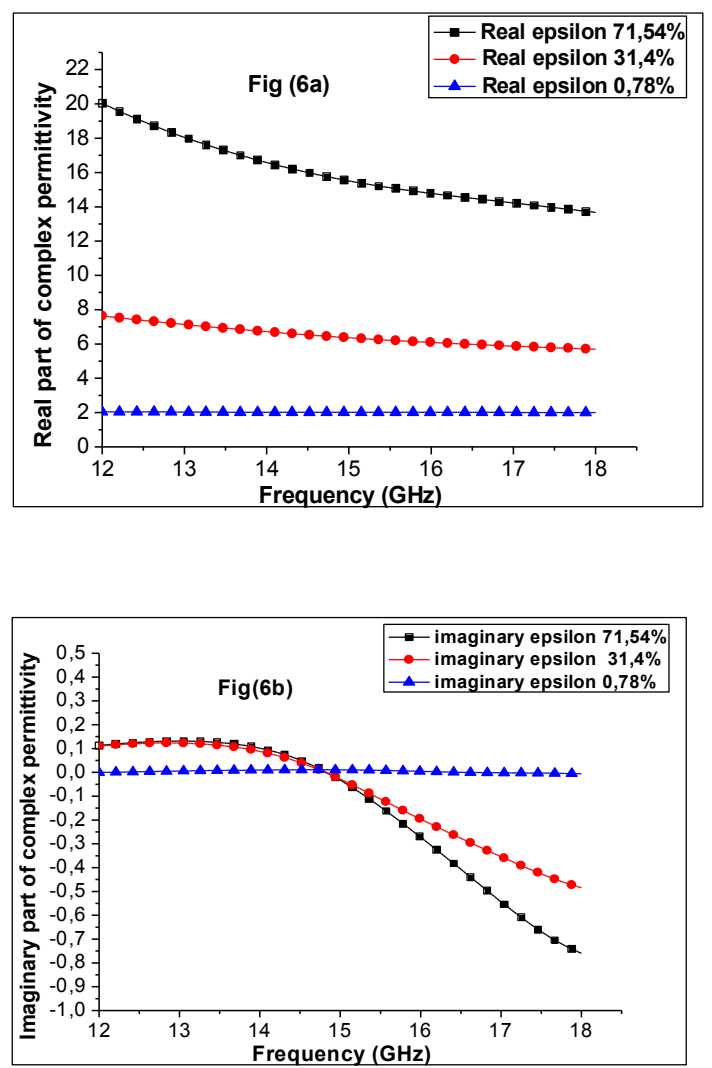

Fig. 6. Evolution of the real (Fig 6a) and imaginary (Fig 6b) parts of the complex permittivity of the composite with $\mathrm{SCS}_{6} \mathrm{SiC}$ fiber as a function of the frequency for three volume fractions. 
The results show that the values of the real parts $\varepsilon^{\prime}$ and imaginary parts $\varepsilon^{\prime \prime}$ of the complex permittivity are generally high due to the high volume fraction $71.54 \%$, and consequently, the decrease in dielectric losses for composites with high volume fraction. This indicates that the filling rate effectively improves the complex permittivity parameters of the composite material. As the volume fraction increases, more mobile charges are produced at the interfaces, leading to an improvement of the electronic polarization and relaxation polarization [4]. Therefore, the real part of the permittivity increases with the increase of the volume fraction.

In the microwave frequency range, the imaginary part of the permittivity is determined both by the relaxation loss of polarization and by the relaxation loss of conduction [15]. If the volume fraction increases, the conductivity increases significantly, leading to an increase in conduction loss. The relaxation polarization is also reinforced, resulting in an increase in the corresponding relaxation loss and improvement of the imaginary part of the complex permittivity. These results also indicate that the evolution of the complex permittivity of the two samples studied depends on the frequency. The curves (5a) and (6a) of the real parts of the permittivity for the two composites in the $\mathrm{Ku}$ band are decreasing as a function of the frequency, as well as the values of the imaginary part. The latter takes negative values when the frequency increases from $12 \mathrm{GHz}$ to $18 \mathrm{GHz}$. The results obtained in this work indicate the existence of dielectric losses in the composites studied for the high frequencies. This behavior is due to the relaxation losses of the polarization by the hysteresis between the displacement current and the accumulation potential when the frequency increases $[1,16]$.

\section{Conclusion}

In this study, we adopted the transmission/reflection method to determine the complex permittivity of two ceramic composites with Nicalon $\mathrm{SiC}$ fiber and SCS6SiC fiber in the Ku-band. The Sij parameters are extracted by rectangular waveguide. Using the Nicholson-Ross-Weir (NRW) algorithm, we determined the complex permittivity of the composites studied.Composites with a high volume fraction have a higher permittivity than others. The results obtained with this method prove that the real and imaginary parts of the complex permittivity decrease simultaneously with increasing frequency in the range of $12 \mathrm{GHz}$ to $18 \mathrm{GHz}$. In addition, the imaginary part takes negative values. These results affirm that $\mathrm{SiC}$ ceramic matrix composite reinforced with $\mathrm{SiC}$ fibers can produce composites with interesting dielectric properties and good microwave absorbers.

\section{References}

[1] Y. Ouhassan, S. Bri, M. C .Elboubakraoui, and M. Habibi. ,FME Trans, 48, 4, 908-913 (2020)
[2] N. Jebbor, S. Bri, A.M. Sánchez, M. Chaibi., Meas, 46, 2206-2209 (2013)

[3] L.P .Adriano, C.R. Mirabel, J.B. Joaquim., J. Aerosp .Technol. Manag. São José dos Campos, 3, 1,59-64(2011)

[4] Y. Ouhassan, S. Bri, M. Habibi, IJATCSE, 9, 1.5, 147-153 (2020)

[5] L. Porte, A. Sartre,J. Mater. Sci, 24, 271- 275 (1989)

[6] M. C. El boubakraoui, S. Bri, and J.Foshi, FME Trans, 46, 86-92 (2018)

[7] V.M. Petrov, and V.V .Gagulin, Inorg. Mater, 37, 2, 93-98 (2001)

[8] M. Charles, and N. Ahmad Hawad, 18th International Congress of Metrology, 07010 (2017)

[9] A. M. Nicolson and G. Ross, IEEE Trans. Instrum. Meas, 19, 4, 377-382 (1970)

[10] X. Zhao, Y. Wu, Z. Fan, F.Li, J. Appl. Phys, 95, 12, 8110-8117 (2004)

[11] K.H. Mabhouti, M. Karamirad, P. Norouzzadeh, M.M. Golzan, and R. Naderali, J. Electron. Mater, 49, 3668-3676(2020)

[12] M. C. Elboubakraoui, S. Bri, and J. Foshi, FME Trans, 45, 4, 678-683 (2017)

[13] W.B. Weir, In Proceedings IEEE, 62, 1, 33-36 (1974)

[14] F.X. Qin and H-X. Peng, Prog. Mater Sci, 58, 183-259 (2013)

[15] Y. Wang, F. Lou, W.C. Zhou, and D.M.Zhu, J. Electron. Mater, 46, 8 (2017)

[16] T. Han, R. Luo, G. Cui, L. Wang, J. Eur. Ceram. Soc, 39, 1743-1756 (2019) 